\title{
Effects of Occlusal Forms on Pressure and Bending During Mastication with Complete Dentures
}

\author{
C. KELSEY, J. COPLOWITZ, and M. SCHOONMAKER \\ Complete Denture Department, School of Dentistry, University of Michigan, \\ Ann Arbor, Michigan 48104, USA
}

The amount of strain in functioning dentures (and the resultant tissue distortion) caused by using various posterior teeth of different occlusal configurations has not been satisfactorily investigated. In an attempt to determine which occlusal form produces the greater overall strain and the greater strain per unit pressure applied, interchangeable posterior teeth (zero degree and anatomic) $^{a}$ were adapted to the same maxillary and mandibular cast metal denture bases to keep all denture-related factors constant, except for changes in the occlusal patterns. A diaphragm pressure transducer, calibrated hydrostatically, was mounted in the cast gold base of the mandibular denture under the first molar on the side preferred by the participant for chewing, the diaphragm of the transducer being fitted so that it was flush with the tissue surface of the denture.

Pairs of strain gauges ${ }^{b}$ were mounted on the lingual and buccal flanges of the mandibular denture and on the buccal flange of the maxillary denture on the participant's preferred chewing side. Each pair of gauges was mounted in the area of the first molar and oriented so that the longitudinal strain along the denture axis was measured directly. The second gauge measured strain at an angle of $90^{\circ}$ to the first and was called the vertical strain even though the plane defined by the face of the denture to which the gauges were attached was not a true vertical plane. All wires were embedded in the denture bases and exited between the central incisors to avoid interfering with function.

The participant masticated for a total of 80 sequences; 40 with zero degree teeth and 40 with anatomic teeth. The sequences with each tooth form were separated equally between the test

This investigation was supported by USPHS Research Grant No. 5501 and RR 05321 from the General Research Service Branch, Division of Research Facilities and Resources.

Received for publication March 25, 1975.

Accepted for publication July 29, 1975 .

a Trubyte Rational Posteriors and Trubyte Pilkington. Turner $30^{\circ}$ Posteriors, Dentsply International, York, Penn.

b BAE-I 3-031TC-120, William T. Beam, Inc, Detroit, Mich. foods: raisins and carrots. Each sequence consisted of allowing the participant to masticate the test food until he swallowed voluntarily.

The pressure data were digitized during playback using a digital converter ${ }^{c}$ at a sampling rate of 30 hertz and the average pressure during each masticatory sequence was obtained. The bending data were then analyzed by measuring the height of each peak corresponding to a pressure peak on the records obtained from an eightchannel recorder ${ }^{d}$ using the $X-Y$ digitizer. ${ }^{e}$ These numbers were then averaged for each masticatory sequence to obtain the average peak bending during a sequence.

An analysis of variance was performed on the average pressure and the average peak bending for each food. An analysis of covariance was performed for each food where the effects of the covariate-pressure-on bending were investigated. It was assumed that the strains measured were linear functions of pressure.

An analysis of variance indicated that at a 0.05 level of significance, the average pressure during a masticatory sequence was greater with zero degree teeth for both test foods (carrots, $8 \%$; raisins, $9 \%$ ). Raisins yielded a greater average pressure than carrots for both tooth forms.

Using uncorrected bending data in an analysis of variance, a significant difference at the 0.05 level was found for the buccal longitudinal channel only. When an analysis of covariance was performed in order to correct the average peak bending for the effects of average pressure, the correction increased the average peak bending for anatomic teeth and decreased it for zero degree teeth. The corrected means were significantly greater at the 0.05 level in three of the six cases.

The data indicate that for the test participant, there was significantly greater strain per unit pressure applied for the anatomic (cusp) tooth than for the zero degree (flat) tooth.

\footnotetext{
- Model AF0l-A A/D, Digital Equipment Corporation, Maynard, Mass.

d Brush Mark 480 Eight Channel Recorder, Gould Inc., Brush Instruments Div., Cleveland, Ohio.

- Model PF-40 Digitizer, Edwin Industries Corporation, Maynard, Mass.
} 